\title{
Jordan Type Inequalities for Hyperbolic Functions and Their Applications
}

\author{
Zhen-Hang Yang and Yu-Ming Chu \\ School of Mathematics and Computation Sciences, Hunan City University, Yiyang 413000, China \\ Correspondence should be addressed to Yu-Ming Chu; chuyuming2005@126.com
}

Received 3 August 2014; Accepted 2 September 2014

Academic Editor: Kehe Zhu

Copyright (c) 2015 Z.-H. Yang and Y.-M. Chu. This is an open access article distributed under the Creative Commons Attribution License, which permits unrestricted use, distribution, and reproduction in any medium, provided the original work is properly cited.

We present the best possible parameters $p, q \in(0, \infty)$ such that the double inequality $\left(1 / 3 p^{2}\right) \cosh (p x)+1-1 / 3 p^{2}<\sinh (x) / x<$ $\left(1 / 3 q^{2}\right) \cosh (q x)+1-1 / 3 q^{2}$ holds for all $x \in(0, \infty)$. As applications, some new inequalities for certain special function and bivariate means are found.

\section{Introduction}

The well known Jordan inequality [1] is given by

$$
\frac{2}{\pi} x<\sin (x)<x, \quad x \in\left(0, \frac{\pi}{2}\right) .
$$

During the past few years, the improvements, refinements, and generalizations for inequality (1) have attracted the attention of many researchers [2-13]. Recently, the hyperbolic counterpart and its generalizations have been the subject of intensive research.

Zhu [14] proved that the inequality

$$
\left(\frac{\sinh (x)}{x}\right)^{q}>p+(1-p) \cosh (x)
$$

holds for all $x>0$ if and only if $q \geq 3(1-p)$ if $p \in$ $(-\infty, 8 / 15] \cup(1, \infty)$.

In $[3,15]$, Neuman and Sándor proved that

$$
\cosh ^{4 / 3}\left(\frac{x}{2}\right)<\frac{\sinh (x)}{x}<\cosh ^{3}(x)
$$

for all $x>0$.

Klén et al. [5] proved that the double inequality

$$
\cosh ^{1 / 4}(x)<\frac{\sinh (x)}{x}<\cosh ^{1 / 2}(x)
$$

holds for all $x \in(0,1)$.
In [4], the authors proved that the double inequality

$$
\cosh ^{p}(x)<\frac{\sinh (x)}{x}<\cosh ^{q}(x)
$$

holds for all $x \in(0,1)$ if and only if $p \leq 1 / 3$ and $q \geq$ $[\log (\sinh (1))] /[\log (\cosh (1))]=0.3721 \cdots$.

Zhu $[16,17]$ proved that the inequalities

$$
\begin{aligned}
(1-\lambda)+\lambda \cosh ^{p}(x) & <\left(\frac{\sinh (x)}{x}\right)^{p} \\
& <(1-\mu)+\mu \cosh ^{p}(x), \\
\left(\frac{\sinh (x)}{x}\right)^{q}< & (1-\eta)+\eta \cosh ^{q}(x), \\
\alpha+(1-\alpha) e^{r[t \operatorname{coth}(t)-1]} & <\left(\frac{\sinh (x)}{x}\right)^{r} \\
& <\beta+(1-\beta) e^{r[t \operatorname{coth}(t)-1]}
\end{aligned}
$$

hold for all $x>0$ if and only if $\lambda \leq 0, \mu \geq 1 / 3, \eta \leq 1 / 3, \alpha \geq$ 1 , and $\beta \leq 1 / 2$ if $p \geq 4 / 5, q<0$, and $r \geq 286 / 693$.

Very recently, Yang [18] proved that the double inequality

$$
[\cosh (p x)]^{1 / 3 p^{2}}<\frac{\sinh (x)}{x}<[\cosh (q x)]^{1 / 3 q^{2}}
$$

holds for all $x>0$ if and only if $p \geq \sqrt{5} / 5$ and $q \leq 1 / 3$. 
The main purpose of this paper is to find the best possible parameters $p, q \in(0, \infty)$ such that the double inequality $\left(1 / 3 p^{2}\right) \cosh (p x)+1-1 / 3 p^{2}<\sinh (x) / x<$ $\left(1 / 3 q^{2}\right) \cosh (q x)+1-1 / 3 q^{2}$ holds for all $x>0$ and present several new inequalities for certain special function and bivariate means.

\section{Main Result}

Theorem 1. Let $p, q \in(0, \infty)$. Then the double inequality

$$
\begin{aligned}
\frac{1}{3 p^{2}} \cosh (p x)+1-\frac{1}{3 p^{2}} & <\frac{\sinh (x)}{x} \\
& <\frac{1}{3 q^{2}} \cosh (q x)+1-\frac{1}{3 q^{2}}
\end{aligned}
$$

holds for all $x>0$ if and only if $p \leq \sqrt{15} / 5$ and $q \geq 1$.

Proof. Let $\lambda>0$ and let the function $f_{\lambda}$ be defined on $(0, \infty)$ by

$$
f_{\lambda}(x)=\frac{\sinh (x)}{x}-\left(\frac{1}{3 \lambda^{2}} \cosh (\lambda x)+1-\frac{1}{3 \lambda^{2}}\right) .
$$

Then making use of power series expansions and (9) we get

$$
\begin{aligned}
f_{\lambda}(x) & =\sum_{n=0}^{\infty} \frac{x^{2 n}}{(2 n+1) !}-\left(\frac{1}{3 \lambda^{2}} \sum_{n=0}^{\infty} \frac{(\lambda x)^{2 n}}{(2 n) !}+1-\frac{1}{3 \lambda^{2}}\right) \\
& =\sum_{n=2}^{\infty} \frac{3-(2 n+1) \lambda^{2 n-2}}{3(2 n+1) !} x^{2 n} .
\end{aligned}
$$

Let

$$
a_{n}(\lambda)=3-(2 n+1) \lambda^{2 n-2} .
$$

Then

$$
a_{n}(1)=3-(2 n+1)=-2(n-1)<0
$$

for all $n \geq 2$. Consider

$$
\begin{gathered}
a_{2}\left(\frac{\sqrt{15}}{5}\right)=0, \quad a_{3}\left(\frac{\sqrt{15}}{5}\right)=\frac{12}{25}>0, \\
a_{n+1}\left(\frac{\sqrt{15}}{5}\right)-a_{n}\left(\frac{\sqrt{15}}{5}\right)=\frac{4 \times 3^{n-1}}{5^{n}}(n-1)>0
\end{gathered}
$$

for all $n \geq 2$.

It follows from (13) that

$$
a_{2}\left(\frac{\sqrt{15}}{5}\right)=0, \quad a_{n}\left(\frac{\sqrt{15}}{5}\right)>0
$$

for all $n \geq 3$.

Therefore, inequality (8) holds for all $x>0$ with $p=$ $\sqrt{15} / 5$ and $q=1$ follows from (9)-(12) and (14).

Next, we prove that $p \leq \sqrt{15} / 5$ and $q \geq 1$ if inequality (8) holds for all $x>0$.
If the first inequality of (8) holds for all $x>0$, then from (9) and (10) we have

$$
\lim _{x \rightarrow 0^{+}} \frac{f_{p}(x)}{x^{4}}=\frac{3-5 p^{2}}{360} \geq 0
$$

and $p \leq \sqrt{15} / 5$.

If the second inequality of (8) holds for all $x>0$, then it follows from (9) that

$$
\begin{aligned}
\lim _{x \rightarrow+\infty} \frac{f_{q}(x)}{e^{q x}}= & \frac{1-e^{-2 x}}{2 x} e^{(1-q) x}-\frac{1+e^{-2 q x}}{6 q^{2}} \\
& -\left(1-\frac{1}{3 q^{2}}\right) e^{-q x} \leq 0 .
\end{aligned}
$$

We clearly see that $\lim _{x \rightarrow+\infty}\left(f_{q}(x) / e^{q x}\right)=+\infty$ if $q<1$. Therefore, $q \geq 1$ follows from (16).

Remark 2. It is not difficult to verify that the bound

$$
g_{p}(x)=\frac{1}{3 p^{2}} \cosh (p x)+1-\frac{1}{3 p^{2}}
$$

given in Theorem 1 is strictly increasing with respect to $p$ on $(0, \infty)$ for fixed $x \in(0, \infty)$.

Remark 3. Let $p=\sqrt{15} / 5>3 / 4>\sqrt{2} / 2>2 / 3>\sqrt{3} / 3$ and $q=1<2 \sqrt{3} / 3$. Then Theorem 1 and Remark 2 lead to

$$
\begin{aligned}
\cosh \left(\frac{\sqrt{3} x}{3}\right)< & \frac{3}{4} \cosh \left(\frac{2 x}{3}\right)+\frac{1}{4}<\frac{2}{3} \cosh \left(\frac{\sqrt{2} x}{2}\right)+\frac{1}{3} \\
& <\frac{16}{27} \cosh \left(\frac{3 x}{4}\right)+\frac{11}{27}<\frac{5}{9} \cosh \left(\frac{\sqrt{15} x}{5}\right) \\
& +\frac{4}{9}<\frac{\sinh (x)}{x} \\
& <\frac{1}{3} \cosh (x)+\frac{2}{3}<\frac{1}{2} \cosh ^{2}\left(\frac{\sqrt{3} x}{3}\right)+\frac{1}{2}
\end{aligned}
$$

for all $x>0$.

\section{Applications}

It is well known that

$$
\int_{0}^{\infty} \frac{x}{\sinh (x)}=\frac{1}{2} \psi^{\prime}\left(\frac{1}{2}\right),
$$

where $\psi^{\prime}$ is the trigamma function defined by

$$
\psi^{\prime}(x)=\int_{0}^{\infty} \frac{t e^{-x t}}{1-e^{-t}} d t .
$$

Let

$$
\operatorname{Sh}(x)=\int_{0}^{x} \frac{t}{\sinh (t)} d t .
$$


Then Remark 3 leads to

$$
\int_{0}^{x} \frac{3}{\cosh (t)+2} d t<\operatorname{Sh}(x)<\int_{0}^{x} \frac{9}{5 \cosh (\sqrt{15} t / 5)+4} d t
$$

for all $x>0$.

From (19) and (22) we get the following.

Remark 4. For all $x>0$ one has

$$
\begin{aligned}
\sqrt{3} \log \left(\frac{e^{x}+2-\sqrt{3}}{e^{x}+2+\sqrt{3}}\right)+\sqrt{3} \log (2+\sqrt{3}) \\
<\operatorname{Sh}(x)<2 \sqrt{15} \\
\times\left[\arctan \left(\frac{5}{3} e^{\sqrt{15 x} / 5}+\frac{4}{3}\right)-\arctan (3)\right] .
\end{aligned}
$$

In particular, we have

$$
\begin{aligned}
4.5620 \cdots & =2 \sqrt{3} \log (2+\sqrt{3})<\psi^{\prime}\left(\frac{1}{2}\right) \\
& <2 \sqrt{15}[\pi-2 \arctan (3)]=4.9845 \cdots .
\end{aligned}
$$

For $a, b>0$, the Schwab-Borchardt mean $\mathrm{SB}(a, b)$ [19-21] is given by

$$
\begin{aligned}
& \mathrm{SB}(a, b)=\frac{\sqrt{b^{2}-a^{2}}}{\arccos (a / b)}(a<b), \\
& \mathrm{SB}(a, b)=a(a=b), \\
& \mathrm{SB}(a, b)=\frac{\sqrt{a^{2}-b^{2}}}{\cosh ^{-1}(a / b)}(a>b) .
\end{aligned}
$$

Let $a>b$ and let $x=\cosh ^{-1}(a / b)$. Then $\cosh (x)=a / b$ and $\sqrt{a^{2}-b^{2}} / \cosh ^{-1}(a / b)=b \sinh (x) / x$. It follows from Remark 3 and (26) that

$$
\left[\frac{16}{27} \cosh \left(\frac{3 x}{4}\right)+\frac{11}{27}\right] b<\mathrm{SB}(a, b)<\left[\frac{1}{3} \cosh (x)+\frac{2}{3}\right] b \text {. }
$$

Note that

$$
\begin{gathered}
{\left[\frac{1}{3} \cosh (x)+\frac{2}{3}\right] b=\frac{a+2 b}{3},} \\
{\left[\frac{16}{27} \cosh \left(\frac{3 x}{4}\right)+\frac{11}{27}\right] b} \\
=\frac{8 b^{1 / 4}}{27}\left(\sqrt{2(a+b)(2 a-b)^{2}}+2 b^{3 / 2}\right)^{1 / 2}+\frac{11 b}{27} .
\end{gathered}
$$

From (27) and (28) we get the following.

Remark 5. Let $a>b>0$; then the Schwab-Borchardt mean $\mathrm{SB}(a, b)$ satisfies the double inequality

$$
\begin{aligned}
& \frac{8 b^{1 / 4}}{27}\left(\sqrt{2(a+b)(2 a-b)^{2}}+2 b^{3 / 2}\right)^{1 / 2} \\
& +\frac{11 b}{27}<\operatorname{SB}(a, b)<\frac{a+2 b}{3} .
\end{aligned}
$$

Let $p \in \mathbb{R}$ and let $a, b>0$ with $a \neq b$. Then the arithmetic mean $A(a, b)$, logarithmic mean $L(a, b)$, geometric mean $G(a, b)$, and $p$ th power mean $M_{p}(a, b)$ are defined by

$$
\begin{gathered}
A(a, b)=\frac{a+b}{2}, \quad L(a, b)=\frac{b-a}{\log b-\log a}, \\
G(a, b)=\sqrt{a b}, \\
M_{p}(a, b)=\left(\frac{a^{p}+b^{p}}{2}\right)^{1 / p} \quad(p \neq 0), \\
M_{0}(a, b)=\sqrt{a b}=G(a, b) .
\end{gathered}
$$

It is well known that $M_{p}(a, b)$ is continuous and strictly increasing with respect to $p \in \mathbb{R}$ for fixed $a, b>0$ with $a \neq b$; the main properties for the power mean are given in [22]. Recently, the arithmetic, logarithmic, geometric, and power means have been the subject of intensive research. In particular, many remarkable inequalities can be found in the literature [23-35].

Let $x=(1 / 2) \log (a / b)$; then (30) leads to

$$
\frac{\sinh (x)}{x}=\frac{L(a, b)}{G(a, b)}, \quad \cosh (p x)=\left(\frac{M_{p}(a, b)}{G(a, b)}\right)^{p} .
$$

From Theorem 1 and (31) we get the following.

Remark 6. Let $p, q \in(0, \infty)$; then the double inequality

$$
\begin{gathered}
\frac{1}{3 p^{2}} M_{p}^{p}(a, b) G^{1-p}(a, b)+\left(1-\frac{1}{3 p^{2}}\right) G(a, b) \\
<L(a, b)<\frac{1}{3 q^{2}} M_{q}^{q}(a, b) G^{1-q}(a, b) \\
+\left(1-\frac{1}{3 q^{2}}\right) G(a, b)
\end{gathered}
$$

holds for all $a, b>0$ with $a \neq b$ if and only if $p \leq \sqrt{15} / 5$ and $q \geq 1$. In particular, the double inequality

$$
\begin{gathered}
\frac{5}{9} M_{\sqrt{15} / 5}^{\sqrt{15} / 5}(a, b) G^{1-\sqrt{15} / 5}(a, b)+\frac{4}{9} G(a, b) \\
<L(a, b)<\frac{1}{3} A(a, b)+\frac{2}{3} G(a, b)
\end{gathered}
$$

holds for all $a, b>0$ with $a \neq b$.

\section{Conflict of Interests}

The authors declare that there is no conflict of interests regarding the publication of this paper.

\section{Acknowledgments}

This research was supported by the Natural Science Foundation of China under Grants 61374086 and 11171307 and the Natural Science Foundation of Zhejiang Province under Grant LY13A010004. 


\section{References}

[1] D. S. Mitrinović, Analytic Inequalities, Springer, New York, NY, USA, 1970.

[2] F. Qi, L.-H. Cui, and S.-L. Xu, "Some inequalities constructed by Tchebysheff's integral inequality," Mathematical Inequalities and Applications, vol. 2, no. 4, pp. 517-528, 1999.

[3] E. Neuman and J. Sándor, "On some inequalities involving trigonometric and hyperbolic functions with emphasis on the Cusa-Huygens, Wilker, and Huygens inequalities," Mathematical Inequalities \& Applications, vol. 13, no. 4, pp. 715-723, 2010.

[4] Y. Lv, G. Wang, and Y. Chu, "A note on Jordan type inequalities for hyperbolic functions," Applied Mathematics Letters, vol. 25, no. 3, pp. 505-508, 2012.

[5] R. Klén, M. Visuri, and M. Vuorinen, "On Jordan type inequalities for hyperbolic functions," Journal of Inequalities and Applications, vol. 2010, Article ID 362548, 14 pages, 2010.

[6] Z.-H. Yang, "New sharp Jordan type inequalities and their applications," Gulf Journal of Mathematics, vol. 2, no. 1, pp. 110, 2014.

[7] L. Zhu, "A source of inequalities for circular functions," Computers \& Mathematics with Applications, vol. 58, no. 10, pp. 19982004, 2009.

[8] Z.-H. Yang, "Sharp bounds for seiffert mean in terms of weighted power means of arithmetic mean and geometric mean," Mathematical Inequalities and Applications, vol. 17, no. 2, pp. 499-511, 2014.

[9] Z.-H. Yang, Y.-M. Chu, Y.-Q. Song, and Y.-M. Li, "A sharp double inequality for trigonometric functions and its applications," Abstract and Applied Analysis, vol. 2014, Article ID 592085, 9 pages, 2014.

[10] S.-H. Wu and L. Debnath, "A new generalized and sharp version of Jordan's inequality and its applications to the improvement of the Yang Le inequality," Applied Mathematics Letters, vol. 19, no. 12, pp. 1378-1384, 2006.

[11] S.-H. Wu, "Sharpness and generalization of Jordan's inequality and its application," Taiwanese Journal of Mathematics, vol. 12, no. 2, pp. 325-336, 2008.

[12] Z. Yang, "Refinements of a two-sided inequality for trigonometric functions," Journal of Mathematical Inequalities, vol. 7, no. 4, pp. 601-615, 2013.

[13] Z. Yang and Y. Chu, "A note on Jordan, Adamović-Mitrinović, and Cusa Inequalities," Abstract and Applied Analysis, vol. 2014, Article ID 364076, 12 pages, 2014.

[14] L. Zhu, "Generalized Lazarevic's inequality and its applications. II," Journal of Inequalities and Applications, vol. 2009, Article ID 379142, 4 pages, 2009.

[15] E. Neuman and J. Sándor, "Inequalities for hyperbolic functions," Applied Mathematics and Computation, vol. 218, no. 18, pp. 9291-9295, 2012.

[16] L. Zhu, "Inequalities for hyperbolic functions and their applications," Journal of Inequalities and Applications, vol. 2010, Article ID 130821, 10 pages, 2010.

[17] L. Zhu, "New inequalities for hyperbolic functions and their applications," Journal of Inequalities and Applications, vol. 2012, article 303, 9 pages, 2012.

[18] Z.-H. Yang, "New sharp bounds for logarithmic mean and identric mean," Journal of Inequalities and Applications, vol. 2013, article 116, 2013.

[19] E. Neuman and J. Sándor, "On the Schwab-Borchardt mean," Mathematica Pannonica, vol. 14, no. 2, pp. 253-266, 2003.
[20] E. Neuman and J. Sándor, "On the Schwab-Borchardt mean II," Mathematica Pannonica, vol. 17, no. 1, pp. 49-59, 2006.

[21] E. Neuman, "Inequalities for the Schwab-Borchardt mean and their applications," Journal of Mathematical Inequalities, vol. 5, no. 4, pp. 601-609, 2011.

[22] P. S. Bullen, D. S. Mitrinović, and P. M. Vasić, Means and Their Inequalities, vol. 31, D. Reidel Publishing Co., Dordrecht, Netherlands, 1988.

[23] B. C. Carlson, "The logarithmic mean," The American Mathematical Monthly, vol. 79, pp. 615-618, 1972.

[24] T. P. Lin, "The power mean and the logarithmic mean," The American Mathematical Monthly, vol. 81, pp. 879-883, 1974.

[25] J. Sándor, "On the identric and logarithmic means," Aequationes Mathematicae, vol. 40, no. 2-3, pp. 261-270, 1990.

[26] H. Alzer and S. Qiu, "Inequalities for means in two variables," Archiv der Mathematik, vol. 80, no. 2, pp. 201-215, 2003.

[27] Y.-M. Chu and W.-F. Xia, "Two optimal double inequalities between power mean and logarithmic mean," Computers \& Mathematics with Applications, vol. 60, no. 1, pp. 83-89, 2010.

[28] Y. Chu, Y. Qiu, and M. Wang, "Sharp power mean bounds for the combination of Seiffert and geometric means," Abstract and Applied Analysis, vol. 2010, Article ID 108920, 12 pages, 2010.

[29] H. Liu and X.-J. Meng, "The optimal convex combination bounds for Seiffert's mean," Journal of Inequalities and Applications, vol. 2011, Article ID 686834, 9 pages, 2011.

[30] H. Gao, J. Guo, and W. Yu, "Sharp bounds for power mean in terms of generalized Heronian mean," Abstract and Applied Analysis, vol. 2011, Article ID 679201, 9 pages, 2011.

[31] M.-K. Wang, Y.-M. Chu, Y.-F. Qiu, and S.-L. Qiu, "An optimal power mean inequality for the complete elliptic integrals," Applied Mathematics Letters, vol. 24, no. 6, pp. 887-890, 2011.

[32] W.-D. Jiang and F. Qi, "Some sharp inequalities involving Seiffert and other means and their concise proofs," Mathematical Inequalities \& Applications, vol. 15, no. 4, Article ID 15-86, pp. 1007-1017, 2012.

[33] W.-D. Jiang, "Some sharp inequalities involving reciprocals of the Seiffert and other means," Journal of Mathematical Inequalities, vol. 6, no. 4, pp. 593-599, 2012.

[34] Y. M. Chu, M. Y. Shi, and Y. P. Jiang, "Optimal inequalities for the power, harmonic and logarithmic means," Iranian Mathematical Society. Bulletin, vol. 38, no. 3, pp. 597-606, 2012.

[35] Y. M. Chu, S. L. Qiu, and M. K. Wang, "Sharp inequalities involving the power mean and complete elliptic integral of the first kind," The Rocky Mountain Journal of Mathematics, vol. 43, no. 5, pp. 1489-1496, 2013. 


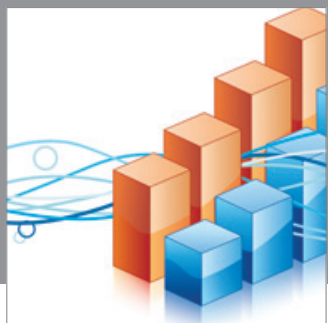

Advances in

Operations Research

mansans

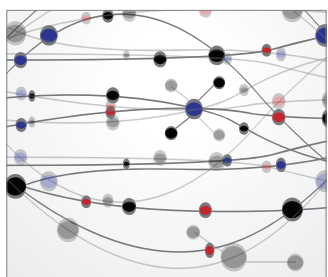

The Scientific World Journal
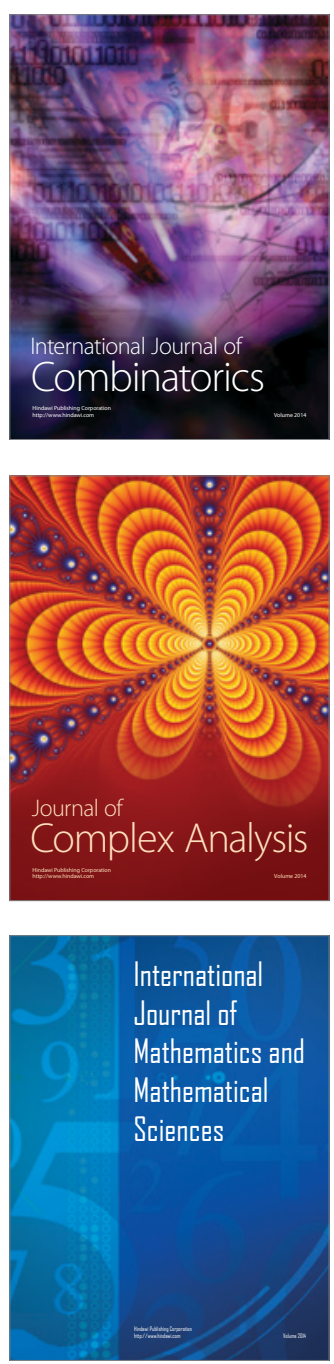
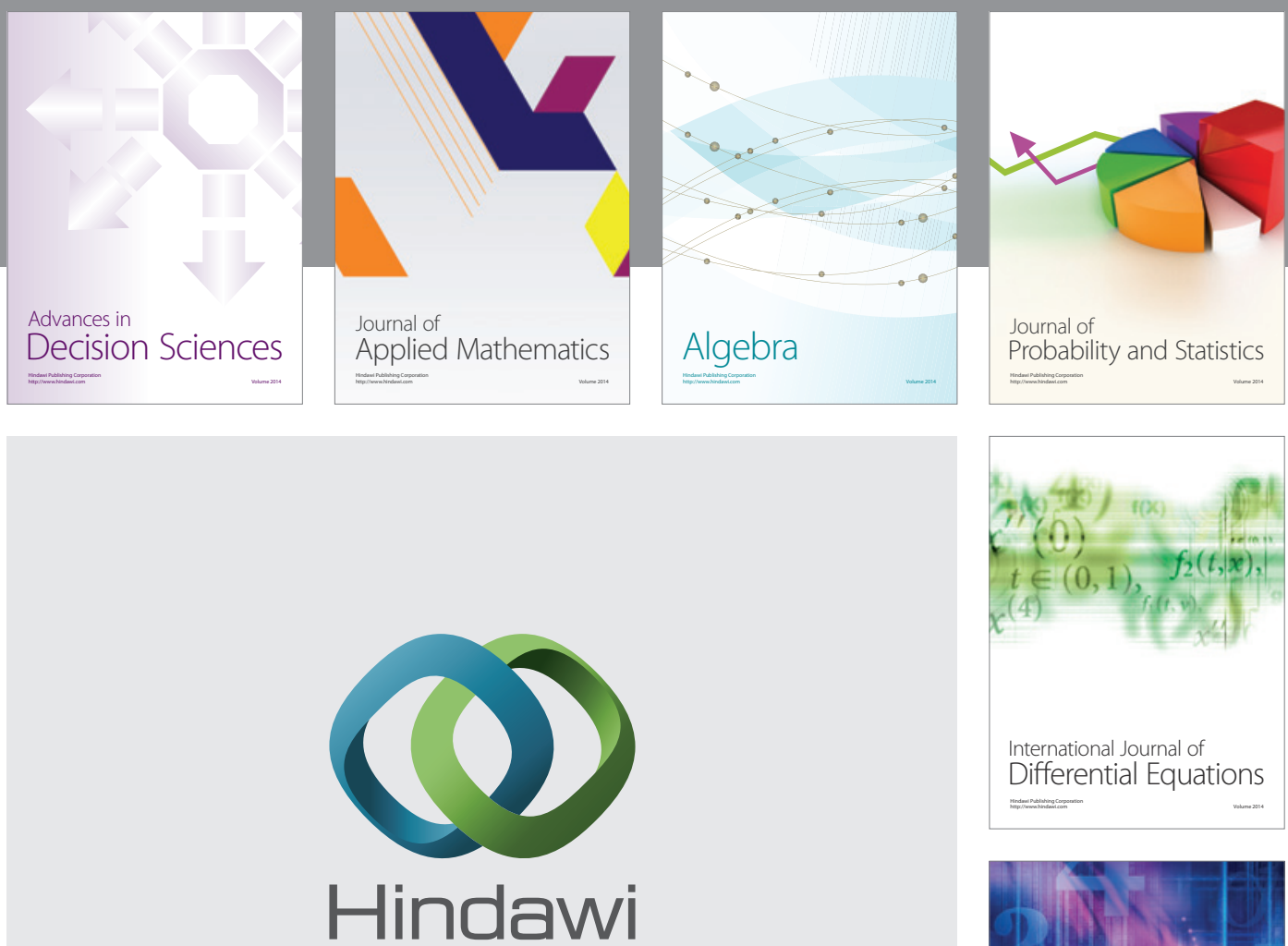

Submit your manuscripts at http://www.hindawi.com
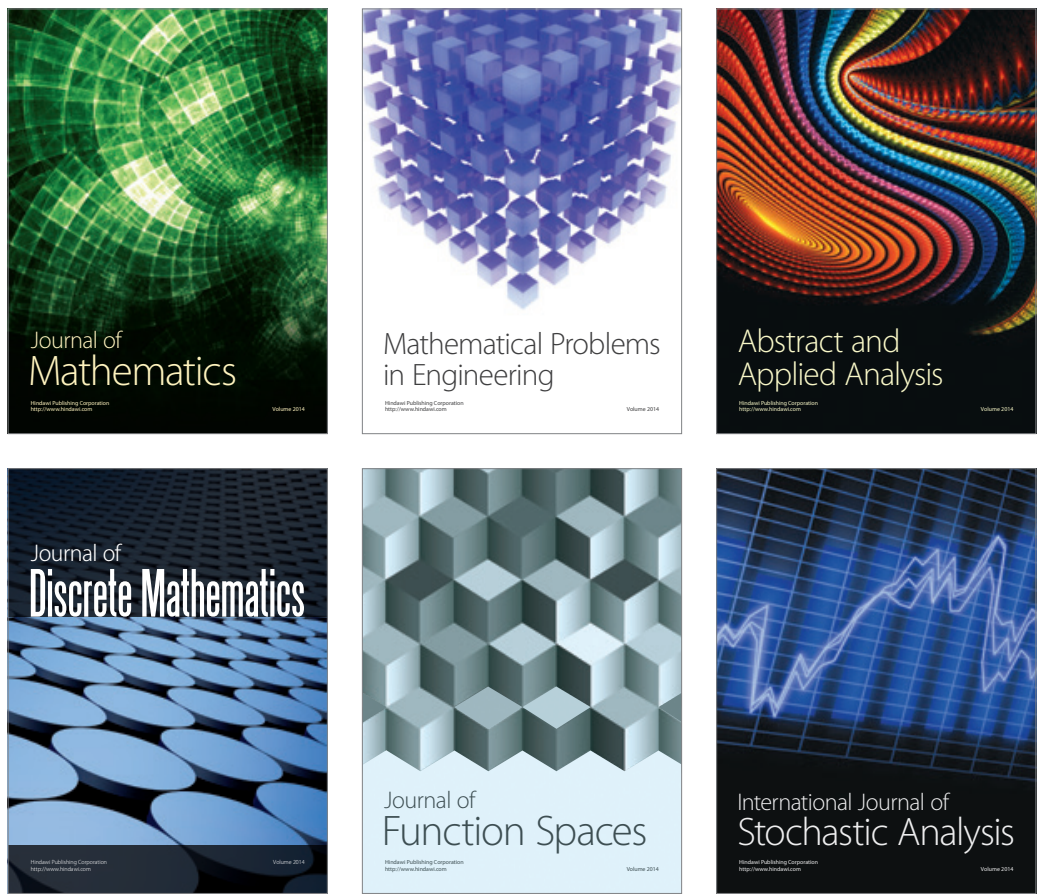

Journal of

Function Spaces

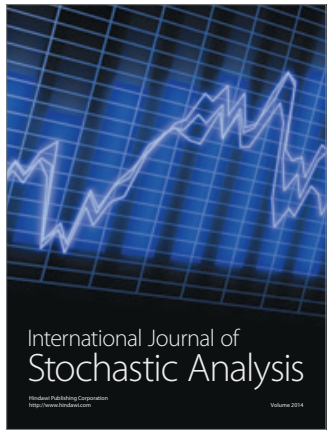

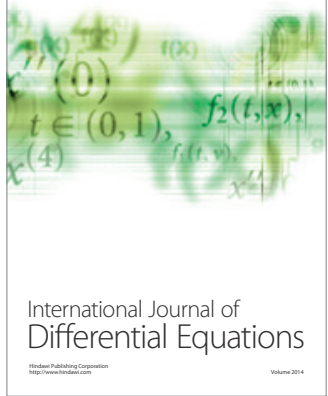
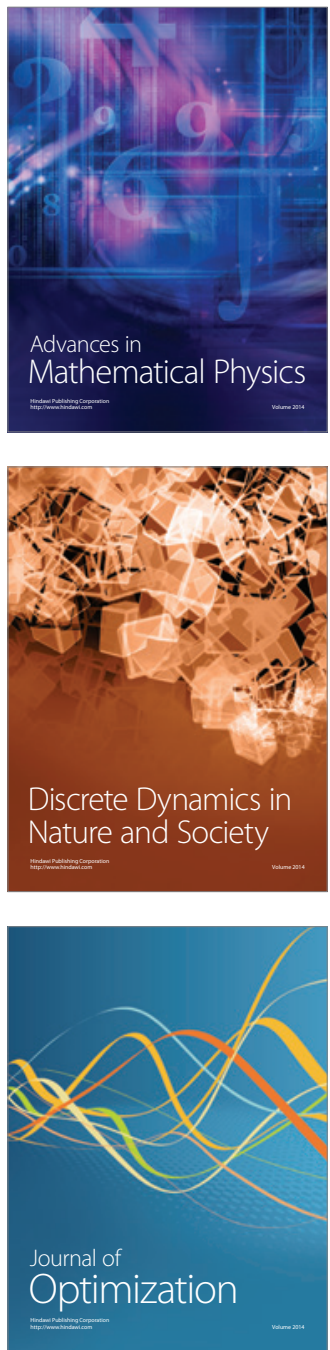